\title{
A special issue of the Australian society for Biophysics
}

\author{
Cristobal dos Remedios ${ }^{1} \cdot$ Charles Cranfield ${ }^{2} \cdot$ Donna Whelan $^{3} \cdot$ Charles Cox $^{1,4} \cdot$ Keith Shearwin $^{5} \cdot$ Joshua Ho $^{6}$. \\ Toby Allen $^{7} \cdot$ Risa Shibuya ${ }^{8} \cdot$ Emi Hibino $^{9} \cdot$ Kumiko Hayashi $^{10} \cdot$ Amy Li $^{3,11}$
}

Received: 1 February 2022 / Accepted: 3 February 2022 / Published online: 23 February 2022

(c) International Union for Pure and Applied Biophysics (IUPAB) and Springer-Verlag GmbH Germany, part of Springer Nature 2022

\begin{abstract}
On behalf of the Australian Society for Biophysics (ASB) and the Editors of this Special Issue, I would like to express our appreciation to Editor-in-Chief, Damien Hall, for arranging the publication of this Special Issue. The ASB is about five times smaller than our sister the Biophysical Society for Japan (BSJ) and tenfold smaller than the US Biophysical Society (USBS), but our meetings are notable because of the encouragement the Society gives to emerging biophysicists. It can be a terrifying experience for a PhD student to have to face a roomful of professors and senior academics, but invariably they appreciate the experience. Another feature of the ASB meetings is the inclusion of contributions from the Asian Pacific region. We now have formal ties with our New Zealand colleagues and our meetings with the BSJ contain joint sessions (see below). In 2020, despite the impact of COVID-19 (see Adam Hill's Commentary), there is a joint session with the University of California Davis. This Special Issue comprises 2 Editorials, 3 Commentaries, and 25 reviews.
\end{abstract}

When we began to put together an editorial on the contributions to this Special Issue of the 44th meeting of the Australian Society for Biophysics (ASB), we were struck by the sheer diversity of what we call "Biophysics". Biophysics is actually not easy to define. The glib answer is "Biophysics is what biophysicists do", but what do they do? If we asked an Australian Minister for Science to tell us what biophysicists do, he or she could tell us what immunologists and virologists do, but would probably have no idea what a biophysicist does. So how should we explain biophysics to the Minister? The US Biophysical Society defines "biophysics"

Cristobal dos Remedios

c.dosremedios@victorchang.edu.au

$\triangle$ Amy Li

Amy.Li@latrobe.edu.au

1 Victor Chang Cardiac Research Institute, Darlinghurst, NSW, Australia

2 School of Life Science, Faculty of Science, University of Technology, Sydney, Ultimo, NSW, Australia

3 Department of Pharmacy and Biomedical Sciences, La Trobe Institute for Molecular Science, La Trobe University, Bendigo, VIC, Australia

4 St Vincent's Clinical School, University of New South Wales, Sydney, NSW, Australia

5 School of Biological Sciences, The University of Adelaide, Adelaide, SA, Australia as the field that applies the theories and methods of physics to understand how biological systems work. Operationally, biophysicists analyse the structure of biological molecules like DNA and proteins, they develop computer models to understand how drugs bind to the receptors in the body, and they investigate how gene mutations change the function of proteins.

We thought a good example of biophysics research is the article by Boris Martinac at the beginning of this Special Issue. Boris has worked for much of his research life on trying to figure out how a mechanosensitive ion channel works.

6 School of Biomedical Sciences, Li Ka Shing Faculty of Medicine, University of Hong Kong, Pok Fu Lam, Hong Kong

7 School of Science, RMIT University, Melbourne, VIC, Australia

8 Biomedical Research Institute, National Institute of Advanced Industrial Science and Technology (AIST), Tsukuba, Ibaraki, Japan

9 Graduate School of Pharmaceutical Sciences, Nagoya University, Nagoya, Aichi, Japan

10 Department of Applied Physics, School of Engineering, Tohoku University, Sendai, Japan

11 Centre for Health Futures, Torrens University, Pyrmont, Australia 
His "babies" are molecules encoded by the $M s c L$ and $M s c S$ genes and more recently also by the Piezol gene. He realised that bacteria needed to have sensors embedded in their surface membrane so they can quickly produce electrical or chemical signals in response to a mechanical force which occurs in the form of osmotic pressure. This of course is what enables the bacterium to survive when exposed to a hypoosmotic shock. More recently he and his colleagues turned their attention to investigating whether Piezo1 channels are the inherently mechanosensitive channels in vertebrates (Syeda et al. 2016) like MscL and MscS channels are in bacteria. They explained how Piezo receptors respond to changes in mechanical curvature of the cell membranes that open non-specific cation channels, thereby generating an electrical signal. In 2013 Boris was elected to the Australian Academy of Science in recognition of his discovery of bacterial mechanosensitive channels and the physical principles of mechanosensitive channel gating. More recently his work has expanded into the roles of mechanosensitive channels in nerves and heart disease. While we all hope he would get the "big" prize in science, it was his colleague, Ardem Patapoutian, who was awarded a share for the 2021 Nobel Prize in Physiology or Medicine for his research on Piezo1 and Piezo2.

The 44th meeting of the Australian Society for Biophysics (ASB) was notable for two other reasons. It was either despite the fact or because it was a virtual meeting that the Society concurrently ran an international symposium with our sister society in Japan the Japanese Society for Biophysics. There is a close connection between the ABS and JSB. For years they have encouraged Australian biophysicists to travel to the large JSB meetings in Japan and they regularly send a strong contingent to Australia. A lot of hard work was put in by Kumiko Hayashi and her colleagues Risa Shibuya and Emi Hibino and the meeting attracted Japanese biophysicists from Tsukuba, Osaka, Kyoto, Shinjuku, Okayama, Kawasaki and Nagoya.

The Society also hosted a virtual Early Career Researcher symposium which involved ASB and the University of California Davis. This was chaired by Dr Adam Hill and we refer you to his Commentary where he writes about the challenges and successes of running a virtual meeting "Biophysics in the time of COVID".

The ASB has had a long-standing policy to encourage presentations from early career biophysicists, even as early as $\mathrm{PhD}$ students. These young biophysicists prepare carefully and seem to enjoy what can be a terrifying experience. Professor Jamie Vandenberg moderated a session on careers in biophysics where participants discussed the latest technology in ultrasound, the Victor Chang Innovation Centre, strategies for careers outside of traditional biophysics, the importance of scientific communication and advocacy, and the importance intellectual property law, and finally, there were some encouraging words on a career in biophysics from Boris Martinac.

Our friends across the "ditch" in New Zealand had a session that discussed calcium imaging in mouse models of disease, the impact fibrosis on Ca signalling, high-content super-resolution microscopy, effects of ryanodine receptor clustering on arrhythmia, the impact of fibrosis on cardiac Ca signalling, how $\mathrm{N}$-glycans affect shear force activation of Na channels, and a fascinating analysis of how insects have managed to adapt their flight muscles to achieve highfrequency flapping flight.

The meeting finished with a presentation of the McAuley-Hope prize for a biophysicist who crosses boundaries in biophysics and develops new techniques and methods. It is not always presented but Dr Till Boecking at the University of New South Wales was the well-deserved winner of this much sought-after Prize.

Data availability Not applicable.

\section{Declarations}

Ethics approval This article does not contain any studies involving participants or animals performed by the authors.

Consent to participate Not applicable.

Consent to publish Not applicable.

Competing interests The authors declare no competing interests.

\section{Reference}

Syeda R, Florendo MN, Cox CD, Kefauver JM, Santos JS, Martinac B, Patapoutian A (2016) Piezo1 channels are inherently mechanosensitive. Cell Rep 17:P1739-1746. https://doi.org/10.1016/j. celrep.2016.10.033

Publisher's note Springer Nature remains neutral with regard to jurisdictional claims in published maps and institutional affiliations. 